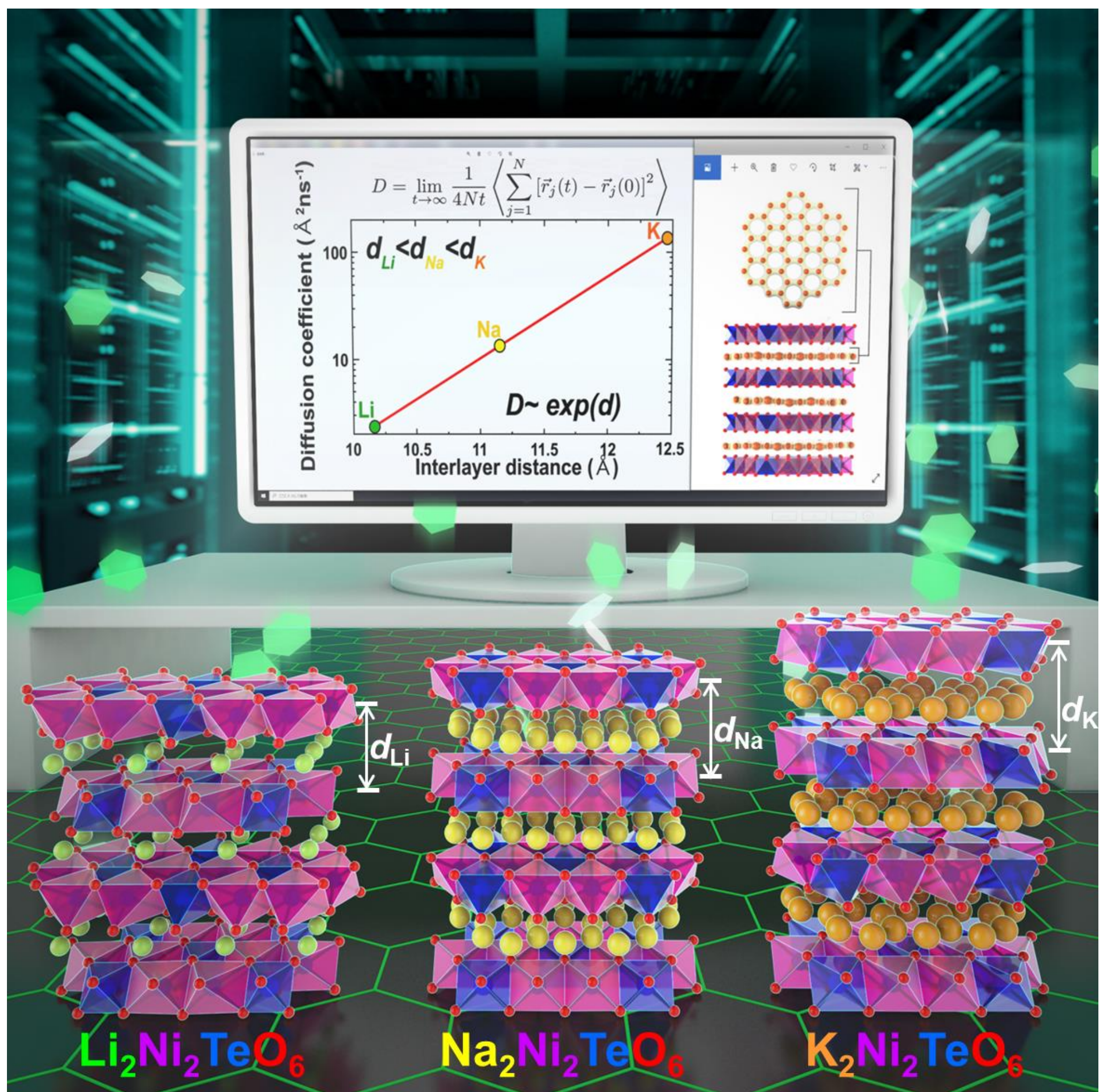

\title{
Role of Interlayer Distance on Cationic Diffusion of Nickel-Based
} Honeycomb Layered Tellurates 
$\operatorname{Kartik~Sau}^{a *}$, Tamio Ikeshoji ${ }^{a}$, Godwill Mbiti Kanyolo $^{b}$, and Titus Masese ${ }^{c, d *}$

${ }^{a}$ Mathematics for Advanced Materials Open Innovation Laboratory (MathAM-OIL), National Institute of Advanced Industrial Science and Technology (AIST), c/o Advanced Institute for Materials Research (AIMR), Tohoku University, Sendai 980-8577, JAPAN

${ }^{b}$ Department of Engineering Science, The University of Electro-Communications, 1-5-1 Chofugaoka, Chofu, Tokyo 182-8585, JAPAN

${ }^{c}$ Research Institute of Electrochemical Energy, National Institute of Advanced Industrial Science and Technology (AIST), 1-8-31 Midorigaoka, Ikeda, Osaka 563-8577, JAPAN

d AIST-Kyoto University Chemical Energy Materials Open Innovation Laboratory (ChEM-OIL), Sakyo-ku, Kyoto 606-8501, JAPAN 


\section{$\underline{\text { Abstract }}$}

Although the fascinatingly rich crystal chemistry of honeycomb layered oxides has been accredited as the propelling force behind their remarkable electrochemistry, the atomistic mechanisms surrounding their operations remain unexplored. Thus, herein, we present an extensive molecular dynamics study performed systematically using a refined set of inter-atomic potential parameters of $A_{2} \mathrm{Ni}_{2} \mathrm{TeO}_{6}$ (where $A=\mathrm{Li}$, Na, and $\mathrm{K}$ ). We demonstrate the effectiveness of the Vashishta-Rahman form of the interatomic potential in reproducing various structural and transport properties of this promising class of materials and predict an exponential increase in cationic diffusion with larger interlayer distances. The simulations further demonstrate the correlation between broadened inter-layer (inter-slab) distances associated with the larger ionic radii of $\mathrm{K}$ and $\mathrm{Na}$ compared to $\mathrm{Li}$ and the enhanced cationic conduction exhibited in $\mathrm{K}_{2} \mathrm{Ni}_{2} \mathrm{TeO}_{6}$ and $\mathrm{Na}_{2} \mathrm{Ni}_{2} \mathrm{TeO}_{6}$ relative to $\mathrm{Li}_{2} \mathrm{Ni}_{2} \mathrm{TeO}_{6}$. Whence, our findings connect a wider bottleneck along the cationic diffusion channel within frameworks comprised of larger mobile cations to the improved cationic diffusion experimentally observed in honeycomb layered oxides.

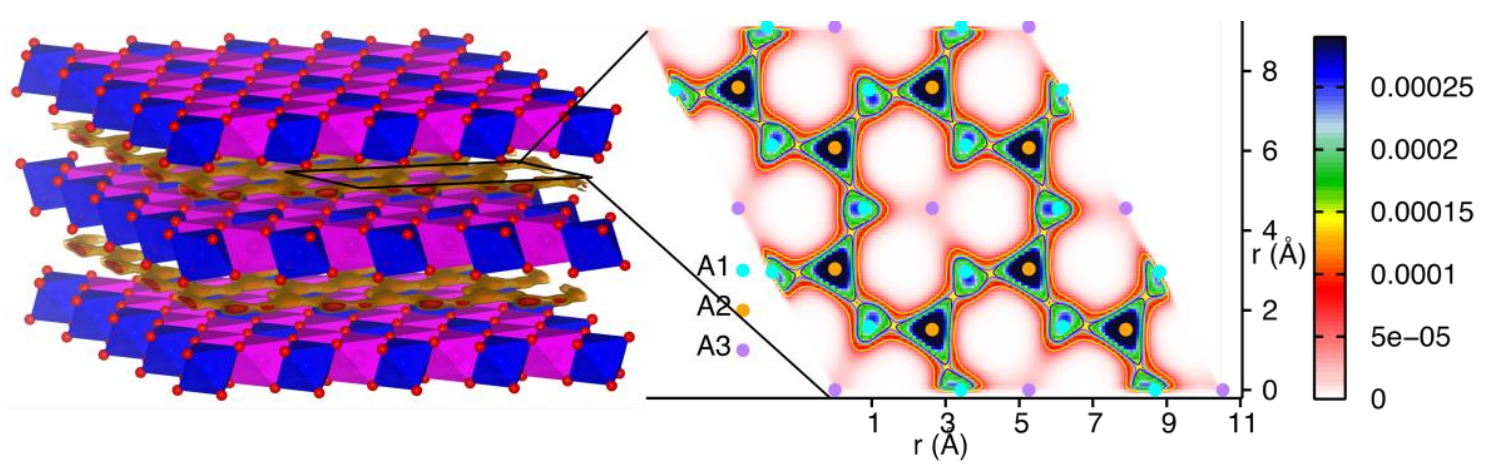

Keywords: Honeycomb layered oxides, cationic diffusion, interlayer distance, molecular dynamics simulations 


\section{INTRODUCTION}

As the winds of change continue to push humanity to the crescendo of an energy revolution, high-energy-density storage systems have gained momentous traction, with lithium-ion batteries at the epicentre of both commercial and industrial applications. ${ }^{\text {[1-3] }}$ This dominance of lithium-ion chemistries is principally propelled by their low redox potential $(-3.04 \mathrm{~V}$ versus standard hydrogen electrode, for instance, in aqueous electrolytes) along with expedient ion kinetics that facilitate the development of layered oxide cathode materials that engender batteries with high voltages, high-power-densities and excellent cyclability. ${ }^{[2,3]}$ However, the sustainability of lithium-ion batteries is heavily impeded by the prohibitive costs, safety issues and scarcity of lithium resources, galvanising explorations into alternative chemistries with terrestrial affluence such as sodium-ion and potassium-ion batteries. ${ }^{[4,5]}$ Besides their terrestrial affluence, the low redox potentials of $\mathrm{Na}$ and $\mathrm{K}(-2.71 \mathrm{~V}$ and $-2.93 \mathrm{~V}$ versus standard hydrogen electrode in aqueous electrolytes, respectively), which earmark their potential as high-energy-density materials, make them prime for future energy storage devices.

The success of high-voltage lithium-ion chemistries have been largely attributed to advancements in layered oxide cathode materials. $\mathrm{LiCoO}_{2}$, the first layered oxide to be investigated for lithium-ion battery cathodes, is characterised by monovalent $\mathrm{Li}^{+}$intercalated between metal slabs comprising trivalent cobalt $\left(\mathrm{Co}^{3+}\right)$ connected to oxygen atoms in an octahedral coordination. ${ }^{[1, i b i d]}$ The significant differences in the sizes and charges of the $\mathrm{Li}^{+}$and transition metal $\mathrm{Co}^{3+}$ ions foster favourable cationic ordering, which facilitates fast two-dimensional $\mathrm{Li}^{+}$diffusion across the lithium plane. Although Li-ion chemistries encompassing the above-mentioned structures have been noted to sustain high average voltages $(3.6 \sim 4.1 \mathrm{~V}), \mathrm{Na}$ and $\mathrm{K}$ analogues $\left(\mathrm{Na}_{x} \mathrm{CoO}_{2}\right.$ and $\mathrm{K}_{x} \mathrm{CoO}_{2}$ (where $\left.x<1\right)$ ) generally display low average voltages $(\sim 3 \mathrm{~V})$ resulting into low-energy densities. This is ascribed to the wide interlayer distances created by the large $\mathrm{Na}$ and $\mathrm{K}$ ionic sizes, ${ }^{[6]}$ which lead to diminished ionicities of the transition metal and oxygen bonds thereby reducing the overall redox voltages of the material.

To ameliorate the electrochemical performance and structural stability of layered oxides, partial substitution of the transition metal species with other transition metal species or highly valent species (such as chalcogens or pnictogens) has been employed. In this pursuit, a unique class of heterostructures known as honeycomb layered oxides has garnered prominence as high-energy-density cathode materials for their exquisite crystal 
structures that engender remarkable ion mobility and high voltages even with $\mathrm{Na}$ and $\mathrm{K}$ chemistries. ${ }^{[7-19]}$ These materials typically adopt chemical compositions of $A_{2} M_{2} D O_{6}$, $A_{3} M_{2} D_{0}$ or $A_{4} M D O_{6}$ (where $M$ can be divalent or trivalent transition or coinage-metal atoms such as $\mathrm{Cr}, \mathrm{Mn}, \mathrm{Fe}, \mathrm{Co}, \mathrm{Ni}, \mathrm{Cu}$ or some combination thereof; $D$ represents pentavalent or hexavalent metal atoms such as $\mathrm{Sb}, \mathrm{Te}, \mathrm{Bi}$; and $A$ can be alkali atoms such as $\mathrm{Li}, \mathrm{Na}, \mathrm{K}$, etc. ${ }^{[7]}$ Given the differences in their ionic sizes and valency states, the $2: 1$ atomic ratio of $M$ and $D$ (such as in $A_{2} M_{2} D_{6}$ and $A_{3} M_{2} D_{6}$ ), renders an array of parallel transition metal slabs with a distinct honeycomb arrangement of multiple $M$ atoms surrounding $D$ atoms in a layered framework of interposed $A$ alkali atoms.

Fast ion kinetics and high voltage capabilities are key prerequisites of any energy material geared towards future capacious battery applications. In this quest, the fine-tuning of the honeycomb layered structures which involves the judicious selection of resident species has been the focal point of numerous studies on this subject. ${ }^{\text {[20-25] }}$ For high capacity, trivalent or divalent metals with high structural stability and high redox voltages (such as $\mathrm{Ni}^{2+} / \mathrm{Ni}^{3+} / \mathrm{Ni}^{4+}$ in $A_{2} \mathrm{Ni}_{2} D \mathrm{O}_{6}, A_{3} \mathrm{Ni}_{2} D \mathrm{O}_{6}$ or $A_{4} \mathrm{Ni} D \mathrm{O}_{6}$ ) are favoured as they allow partial alkali atom occupation without disintegrating. ${ }^{[8-19,26]}$ For high voltages, highly valent pnictogen or chalcogen atoms such as $\mathrm{Te}, \mathrm{Bi}$ and $\mathrm{Sb}$ are employed to lower the covalency of the bond between the oxygen atoms and (di or tri) valent transition metals thereby increasing the energy required for (di or tri) valent cation oxidation. In turn, this induces a large increase in the overall voltage of the battery which can be predicted by the Pauling scale $(\mathrm{Te}>\mathrm{Sb}>\mathrm{Bi}$ ) of electronegativity. Indeed, tellurates used alongside $\mathrm{Ni}^{2+}$ in honeycomb layered oxides have been reported to produce the highest voltages (over $4 \mathrm{~V}$ ) to date. ${ }^{[8-10,12-15,19]}$ Thus, for a comparative study on honeycomb layered oxide electrochemical performance, $\mathrm{Ni}^{2+}$-based honeycomb tellurates $\left(A_{2} \mathrm{Ni}_{2} \mathrm{TeO}_{6}\right)$ present an ideal pedagogical platform as a leverage for insights into the optimisation of related honeycomb layered oxides.

To surmise the mechanisms of honeycomb layered frameworks, a crystal structural illustration of $A_{2} \mathrm{Ni}_{2} \mathrm{TeO}_{6}(A=\mathrm{Li}, \mathrm{Na}$ and $\mathrm{K})$ is furnished in Figure 1. As shown, the $A$ atoms are interspersed between metal slabs consisting of $\mathrm{Ni}$ and $\mathrm{Te}$ in an octahedral coordination with oxygen atoms. The interslab (inter-layer) distances increase in the sequence of $\mathrm{Li}<\mathrm{Na}<\mathrm{K}$ in accord with the Shannon-Prewitt ionic size of the alkali atoms (Note that an ordered $\mathrm{Li}_{2} \mathrm{Ni}_{2} \mathrm{TeO}_{6}$ polymorph ${ }^{[9]}$ is used for ease of comparison with the $\mathrm{Na}$ and $\mathrm{K}$ counterparts). ${ }^{[12,20]}$ As affirmed by previous structural studies on 
honeycomb layered frameworks, cations with smaller ionic radii (such as $\mathrm{Li}^{+}$in $\mathrm{Li}_{2} \mathrm{Ni}_{2} \mathrm{TeO}_{6}$ ) are characterised by alkali planes with smaller interlayer distances (consequently stronger interlayer bonds) which require more energy to facilitate two-dimensional (2D) cation diffusion during (de)insertion processes. ${ }^{[7]}$ On the other hand, resident cations with larger ionic radii (such as $\mathrm{Na}^{+}$and $\mathrm{K}^{+}$in $\mathrm{Na}_{2} \mathrm{Ni}_{2} \mathrm{TeO}_{6}$ and $\mathrm{K}_{2} \mathrm{Ni}_{2} \mathrm{TeO}_{6}$ ) foster weaker interlayer bonds that facilitate facile $2 \mathrm{D}$ cationic diffusion during battery operations. As such, Ni-based honeycomb tellurate compositions comprising $\mathrm{Na}$ and $\mathrm{K}$ have demonstrated faster cationic mobilities relative to their Li-based counterparts, with $\mathrm{K}$ displaying the lowest energy barrier for cationic mobility (viz., activation energy). ${ }^{[27,28]}$

Despite the immense potential envisioned in the experimental and theoretical explorations of honeycomb layered materials, details surrounding the various mesoscopic mechanisms engendering their remarkable electrochemical performance still remain unexplored. In light of this, computational methodologies such as molecular dynamics (MD) simulations present a unique avenue to unveil the microscopic physicochemical properties such as ion transport and energetics at atomic scale, otherwise presently beyond experimental reach. ${ }^{[29]}$ In fact, MD simulations have been shown to accurately depict the structural and transport properties of previously reported energy materials such as our previous works on the physicochemical properties of Na-based honeycomb layered tellurates (i.e., $\left.\mathrm{Na}_{2} \mathrm{M}_{2} \mathrm{TeO}_{6}\right){ }^{[30-32]}$ Moreover, these simulations have been instrumental in garnering insights into the diffusive properties of other materials ${ }^{[33]}$ that have later on been experimentally confirmed (e.g., in $\left.\mathrm{Na}_{2} \mathrm{Ni}_{2} \mathrm{TeO}_{6}\right){ }^{[26,28,30]}$

Thus, in this study we present an extensive MD study systematically performed using a refined set of inter-atomic potential parameters in $A_{2} \mathrm{Ni}_{2} \mathrm{TeO}_{6}$ (where $A=\mathrm{Li}, \mathrm{Na}$, or $\mathrm{K}$ ). The MD simulations demonstrate the effectiveness of the Vashishta-Rahman form of the interatomic potential, ${ }^{[34]}$ reproducing various structural and transport properties of this class of materials. Further, we predict that an increase in the interlayer distance results in improved microscopic transport of cations through diffusion. As such, the larger ionic radii of $\mathrm{K}$ and $\mathrm{Na}$ relative to $\mathrm{Li}$ engenders an exponential enhancement of the cationic conduction of $\mathrm{K}_{2} \mathrm{Ni}_{2} \mathrm{TeO}_{6}$ and $\mathrm{Na}_{2} \mathrm{Ni}_{2} \mathrm{TeO}_{6}$, in comparison with $\mathrm{Li}_{2} \mathrm{Ni}_{2} \mathrm{TeO}_{6}$. Through these investigations, we also connect the weaker interlayer bonds of the larger mobile cations with the oxygen within the frameworks to the improved cationic diffusion experimentally observed in honeycomb layered oxides. 


\section{RESULTS AND DISCUSSION}

\subsection{Framework Structure}

As shown in Figure 1, the $A_{2} \mathrm{Ni}_{2} \mathrm{TeO}_{6}$ compounds, where $A=\mathrm{Li}, \mathrm{Na}$, and $\mathrm{K}$, have a layered structure of $A$ cations sandwiched between transition metal slabs of $\mathrm{TeO}_{6}$ and $\mathrm{NiO}_{6}$ octahedra arrayed in a honeycomb fashion. Experimental reports have shown $\mathrm{Na}_{2} \mathrm{Ni}_{2} \mathrm{TeO}_{6}$ and $\mathrm{K}_{2} \mathrm{Ni}_{2} \mathrm{TeO}_{6}$ to embody hexagonal crystal symmetries, ${ }^{[12,20]}$ whilst $\mathrm{Li}_{2} \mathrm{Ni}_{2} \mathrm{TeO}_{6}$ is known to entail an orthorhombic and monoclinic crystal symmetries contingent on the synthetic protocols. ${ }^{[9]}$ Moreover, all the octahedral layers in the $\mathrm{Na}_{2} \mathrm{Ni}_{2} \mathrm{TeO}_{6}$ and $\mathrm{K}_{2} \mathrm{Ni}_{2} \mathrm{TeO}_{6}$ are seen to be identical, whereas a slight shift between the top and bottom layer around the conduction plane is observed in the case of $\mathrm{Li}_{2} \mathrm{Ni}_{2} \mathrm{TeO}_{6}$ (as shown in Figures 1a, 1b and 1c). The layers are held together along the $c$-axis by Van der Waals forces and by interactions mediated via $A$ atoms occupying their interlayers. It is prudent to mention here that alkali atoms in $\mathrm{Na}_{2} \mathrm{Ni}_{2} \mathrm{TeO}_{6}$ and

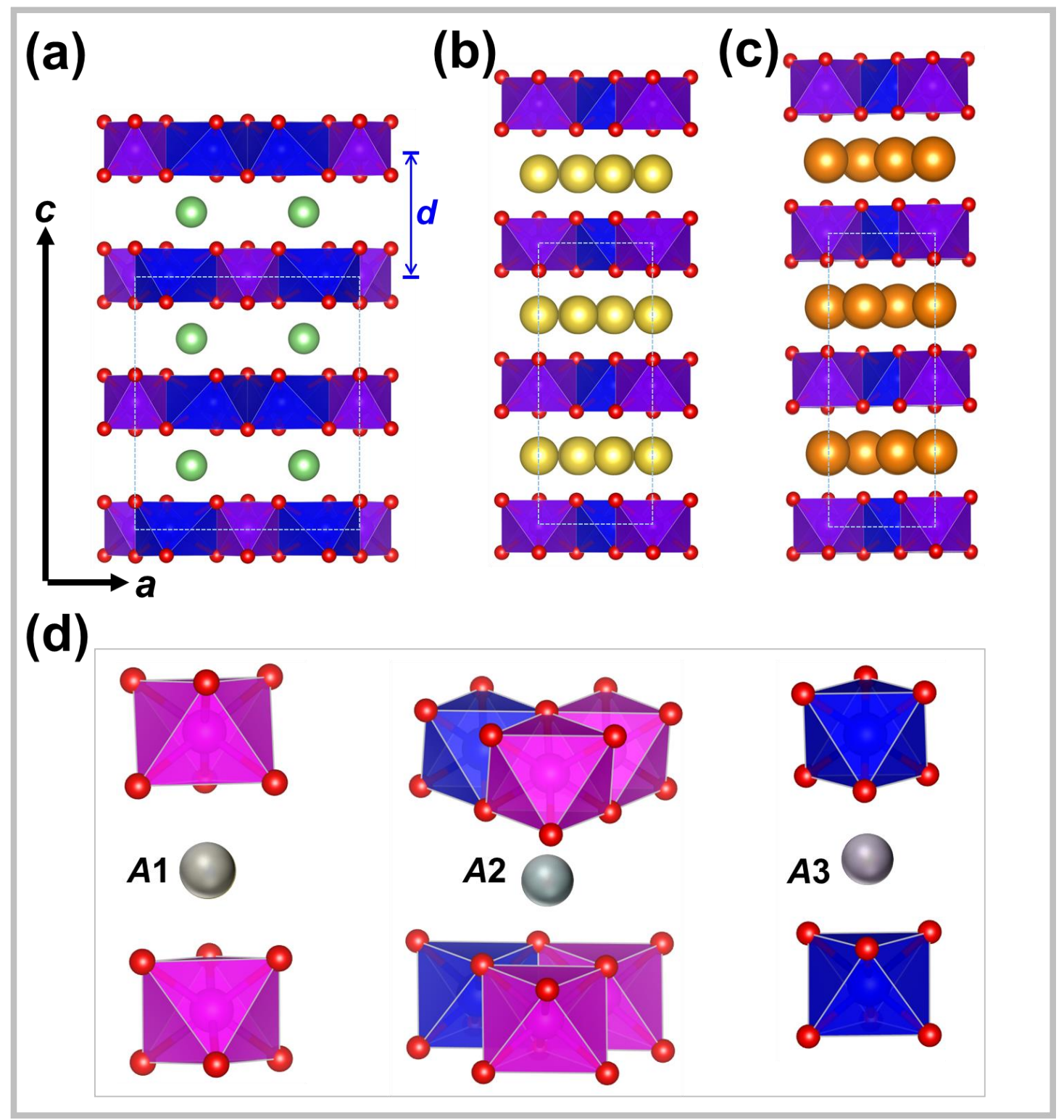


Figure 1. A schematic of the $A_{2} \mathrm{Ni}_{2} \mathrm{TeO}_{6}(A=\mathrm{Li}$, Na and $\mathrm{K})$ crystal structural framework whereby parallel slabs comprising $\mathrm{TeO}_{6}$ octahedra (in blue) and $\mathrm{NiO}_{6}$ octahedra (in magenta) are separated by alkali atoms. (a) Layered structure of $\mathrm{Li}_{2} \mathrm{Ni}_{2} \mathrm{TeO}_{6}$ (orthorhombic polymorph). Li atoms (in green) are located between slabs comprising $\mathrm{Te}$ (in blue) and $\mathrm{Ni}$ (in purple) in octahedral coordination with oxygen atoms (in red). (b) Layered structure of $\mathrm{Na}_{2} \mathrm{Ni}_{2} \mathrm{TeO}_{6}$ where $\mathrm{Na}$ atoms are depicted in yellow. (c) Layered structural framework of $\mathrm{K}_{2} \mathrm{Ni}_{2} \mathrm{TeO}_{6}$ (which is isostructural with $\mathrm{Na}_{2} \mathrm{Ni}_{2} \mathrm{TeO}_{6}$ ). $\mathrm{K}$ atoms are shown in brown. (d) Crystallographically distinct sites where alkali atoms reside in $\mathrm{Na}_{2} \mathrm{Ni}_{2} \mathrm{TeO}_{6}$ or $\mathrm{K}_{2} \mathrm{Ni}_{2} \mathrm{TeO}_{6}$, denoted as $A 1, A 2$ and $A 3$ with partial alkali atom occupation. Their local polyhedral environments are also shown, for clarity. The interlayer (interslab) distance is defined as ' $d$ '. Dashed lines denote the unit cell of $A_{2} \mathrm{Ni}_{2} \mathrm{TeO}_{6}(A=\mathrm{Li}, \mathrm{Na}$ and $\mathrm{K})$ and the axes for the $c$ - and $a$-parameters shown in (a) applies to all the structures displayed.

Table 1. A comparison of average cell parameters (in $\AA$ ) calculated from isobaric-isothermal (NPT) MD simulations performed at $300 \mathrm{~K}$ for the $A_{2} \mathrm{Ni}_{2} \mathrm{TeO}_{6}(A$ $=\mathrm{Li}, \mathrm{Na}$, and $\mathrm{K}$ ) with reported experimental results (abbreviated as 'Exp.').

\begin{tabular}{|c|c|c|c|c|c|c|c|c|c|}
\hline \multirow{2}{*}{$\begin{array}{c}\text { cell } \\
\text { parameters }\end{array}$} & \multicolumn{3}{|c|}{$\mathrm{Li}$} & \multicolumn{3}{|c|}{$\mathrm{Na}$} & \multicolumn{3}{|c|}{$\mathrm{K}$} \\
\hline & Exp. ${ }^{[9]}$ & MD & $\delta$ & Exp. ${ }^{[20]}$ & MD & $\delta$ & Exp. ${ }^{[12]}$ & MD & $\delta$ \\
\hline$a$ & 8.992 & 8.700 & 3.0 & 5.207 & 5.211 & 0.1 & 5.261 & 5.211 & 0.9 \\
\hline$b$ & 5.147 & 4.975 & 3.0 & 5.207 & 5.211 & 0.1 & 5.261 & 5.211 & 0.9 \\
\hline$c$ & 10.169 & 9.829 & 3.0 & 11.1558 & 11.167 & 0.1 & 12.467 & 12.349 & 0.9 \\
\hline
\end{tabular}

$\mathrm{K}_{2} \mathrm{Ni}_{2} \mathrm{TeO}_{6}$ reside in distinct crystallographic sites, denoted as $A 1, A 2$ and $A 3$ with varying occupancy (as succinctly shown in Figure 1d). The refined set of interatomic potential parameters retains these structural features in conformity with experimental findings. The average cell parameters of $A_{2} \mathrm{Ni}_{2} \mathrm{TeO}_{6}$ calculated from NPT-MD 
simulations at $300 \mathrm{~K}$ are placed alongside the room-temperature experimental values in Table 1, where the calculated $c$-parameter (given the crystallographic arrangement of the layers in the $c$-axis direction) increases upon substitution of the $A$ atom species in the order of $\mathrm{Li}, \mathrm{Na}$ and $\mathrm{K}$, respectively, in corroboration with experimental observations. $[9,12,20]$ The progressive increase noted in the cell parameters of this family of materials is ascribed to increases in cation sizes in the order of $\mathrm{Li}, \mathrm{Na}$ and $\mathrm{K}$ as a result of intensified ionic repulsion inside the layers as prescribed in the presence of larger sized cations. It should be noted that the deviations from experimental values in all cases are well within $3 \%$.

For a comparison of the local structures, radial distribution functions, $g(r)$, between the framework ion pairs, $\mathrm{Ni}-\mathrm{O}, \mathrm{Te}-\mathrm{O}$, and $\mathrm{O}-\mathrm{O}$, obtained from NPT-MD simulations at $300 \mathrm{~K}$ for the $A_{2} \mathrm{Ni}_{2} \mathrm{TeO}_{6}$ compounds $(A=\mathrm{Li}, \mathrm{Na}$, and $\mathrm{K}$ ) are furnished in Figure 2. For clarity, the radial distribution functions calculated from the respective X-ray diffraction (XRD) structures ${ }^{[9,12,20,27]}$ are also produced in the form of bar-plots. Except for the thermal broadening, all the calculated peaks from MD simulations are consistent with their corresponding $g(r)$, calculated for the XRD structure. For Te and Ni, exactly six oxygen coordination numbers are found within the $1^{\text {st }}$ neighbouring distance, forming $\mathrm{NiO}_{6}$ and $\mathrm{TeO}_{6}$ octahedra. The average $\mathrm{Te}-\mathrm{O}$ and $\mathrm{Ni}-\mathrm{O}$ distances of the octahedra for $A$ $=\mathrm{Li}, \mathrm{Na}$, and $\mathrm{K}$, calculated from MD simulations (listed in Table 2), also show good agreement with the reported XRD structure. Similarly, the radial distribution functions obtained from the other simulations performed under different conditions (see the METHODOLOGY section) revealed that in all cases, the respective crystal structures were retained, affirming that the refined set of interatomic parameters successfully reproduce the structure. 
(a)

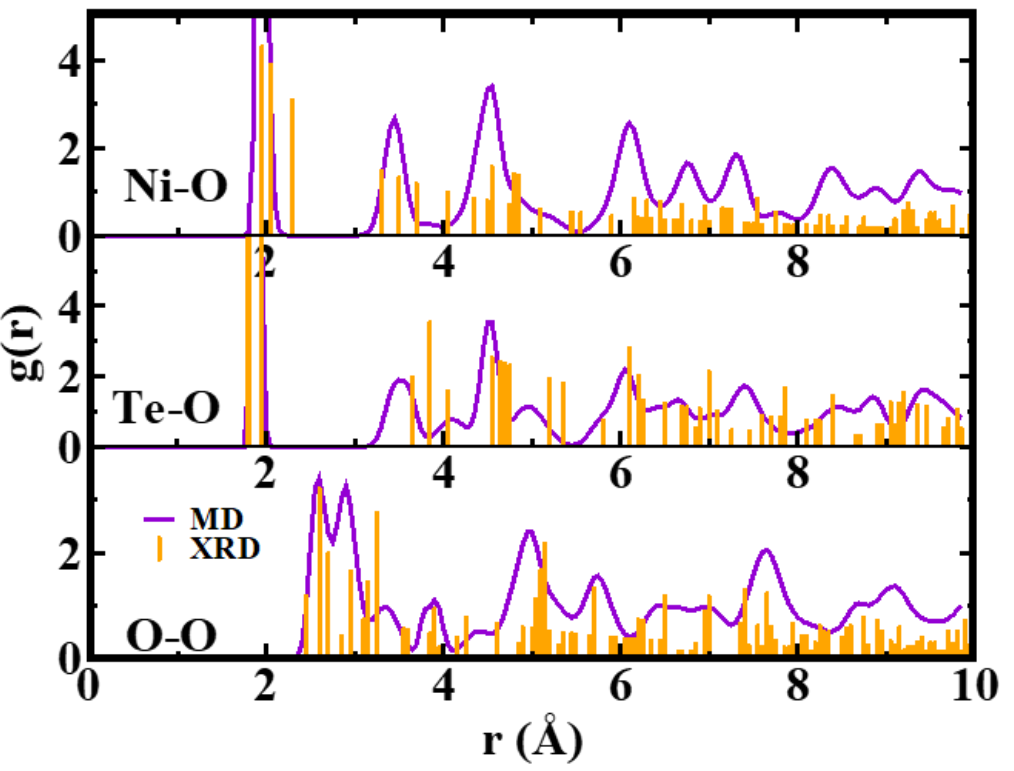

(b)

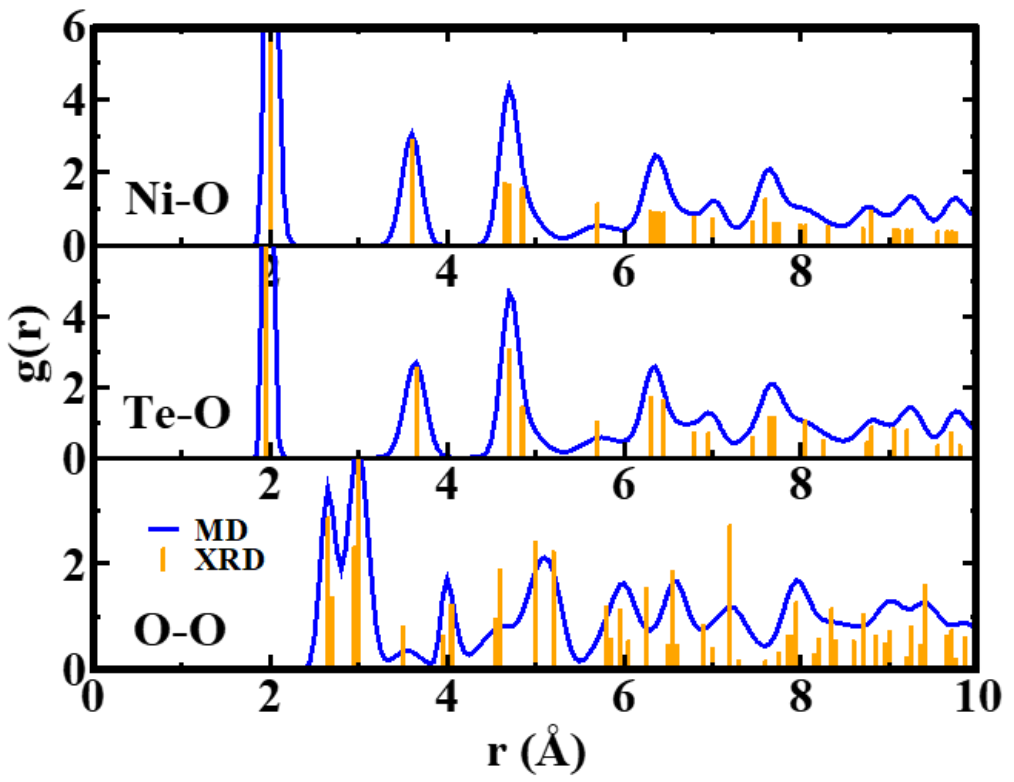

(c)

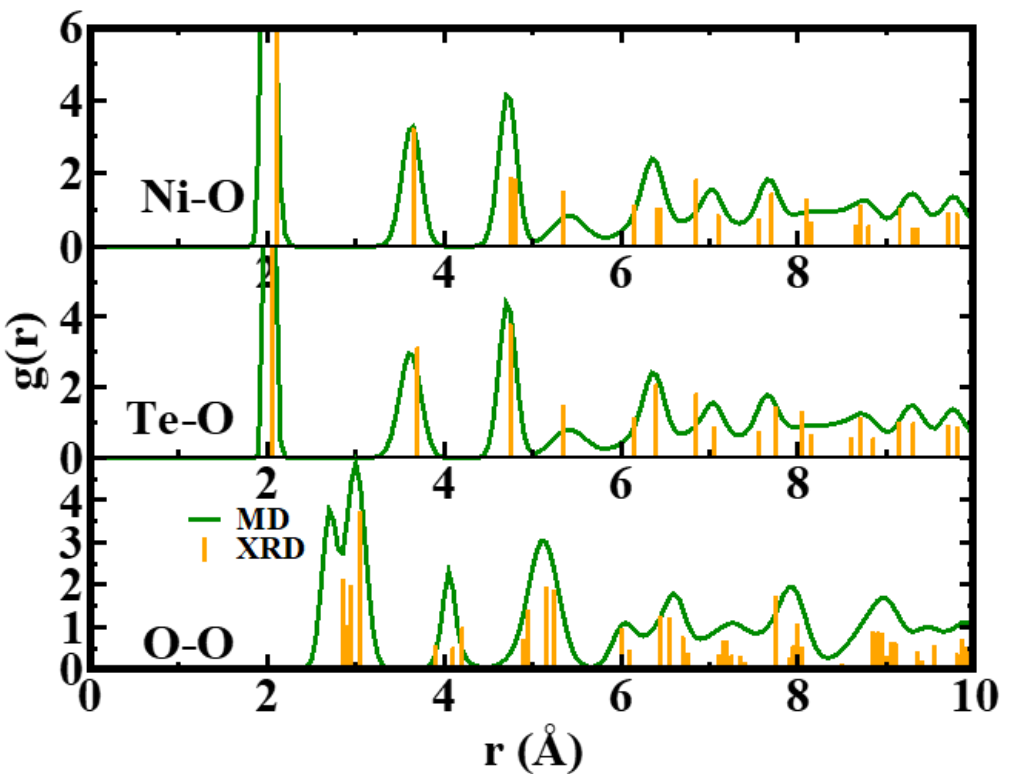

Figure 2. Radial distribution function $(\mathrm{g}(\mathrm{r})$ ) between selected ion pairs (namely, $\mathrm{Ni}-\mathrm{O}$, $\mathrm{Te}-\mathrm{O}$ and $\mathrm{O}-\mathrm{O}$ ) at $300 \mathrm{~K}$ for (a) $\mathrm{Li}_{2} \mathrm{Ni}_{2} \mathrm{TeO}_{6}$, (b) $\mathrm{Na}_{2} \mathrm{Ni}_{2} \mathrm{TeO}_{6}$ and (c) $\mathrm{K}_{2} \mathrm{Ni}_{2} \mathrm{TeO}_{6}$. For clarity to readers, the vertical bars shown in orange are the corresponding radial 
distribution functions derived from X-ray diffraction analyses. ${ }^{[9,12,20,27]}$ The bar heights are reduced for easy visualisation.

Table 2. Average interatomic distances in angstrom $(\AA)$ for the $\mathrm{Te}-\mathrm{O}$ and $\mathrm{Ni}-\mathrm{O}$ octahedra based on NPT-MD simulations conducted at $300 \mathrm{~K}$.

\begin{tabular}{llllllll}
\hline & \multicolumn{2}{c}{ Li } & \multicolumn{3}{c}{$\mathrm{Na}$} & \multicolumn{2}{c}{ K } \\
& Expt. ${ }^{[9]}$ & MD & Expt. ${ }^{[20]}$ & MD & Expt. ${ }^{[12]}$ & MD \\
\hline $\mathrm{Te}-\mathrm{O}$ & 1.993 & 1.990 & 1.971 & 1.990 & 1.951 & 1.990 \\
$\mathrm{Ni}-\mathrm{O}$ & 2.035 & 2.020 & 2.148 & 2.070 & 2.112 & 2.070 \\
\hline
\end{tabular}

\subsection{Ionic Conductivity}

The $A$ cations are located in the $a b$-plane between the polyhedral layers and with loose coordinations to the framework layers which constitute a large number of cationic sublattices. These sublattices facilitate high ionic diffusion, in accordance with equation 2 and ascertained by the mean squared displacements (MSD) of the cations plotted against time (Figure 3(a)). On the other hand, the closely packed octahedral layers parallel to the $a b$-plane restrict the cationic motion along the $c$-axis direction of the cell, as reflected in the inset of Figure 3(a) (less than $0.2 \AA^{2}$ ). Therefore, it can be deduced that cationic mobility is restricted within the sublattices oriented parallel to the $a b$-plane, rendering the diffusion to be highly anisotropic (viz., two-dimensional). The MSD of the $A$ ions is noted to increase drastically with increase in cationic size in the order of $\mathrm{Li}, \mathrm{Na}$ and $\mathrm{K}$. Figure 3(b) displays the diffusion coefficient, which was calculated from the slope of Figure 3(a) using equation 2, as a function of interlayer distance. It is worth noting that the diffusion coefficient unequivocally follows the exponential dependency of the interlayer distance, whereas the experimental activation energy diminishes linearly with increasing interlayer distances as is displayed in inset of Figure 3(b). ${ }^{[20,27]}$ 
(a)
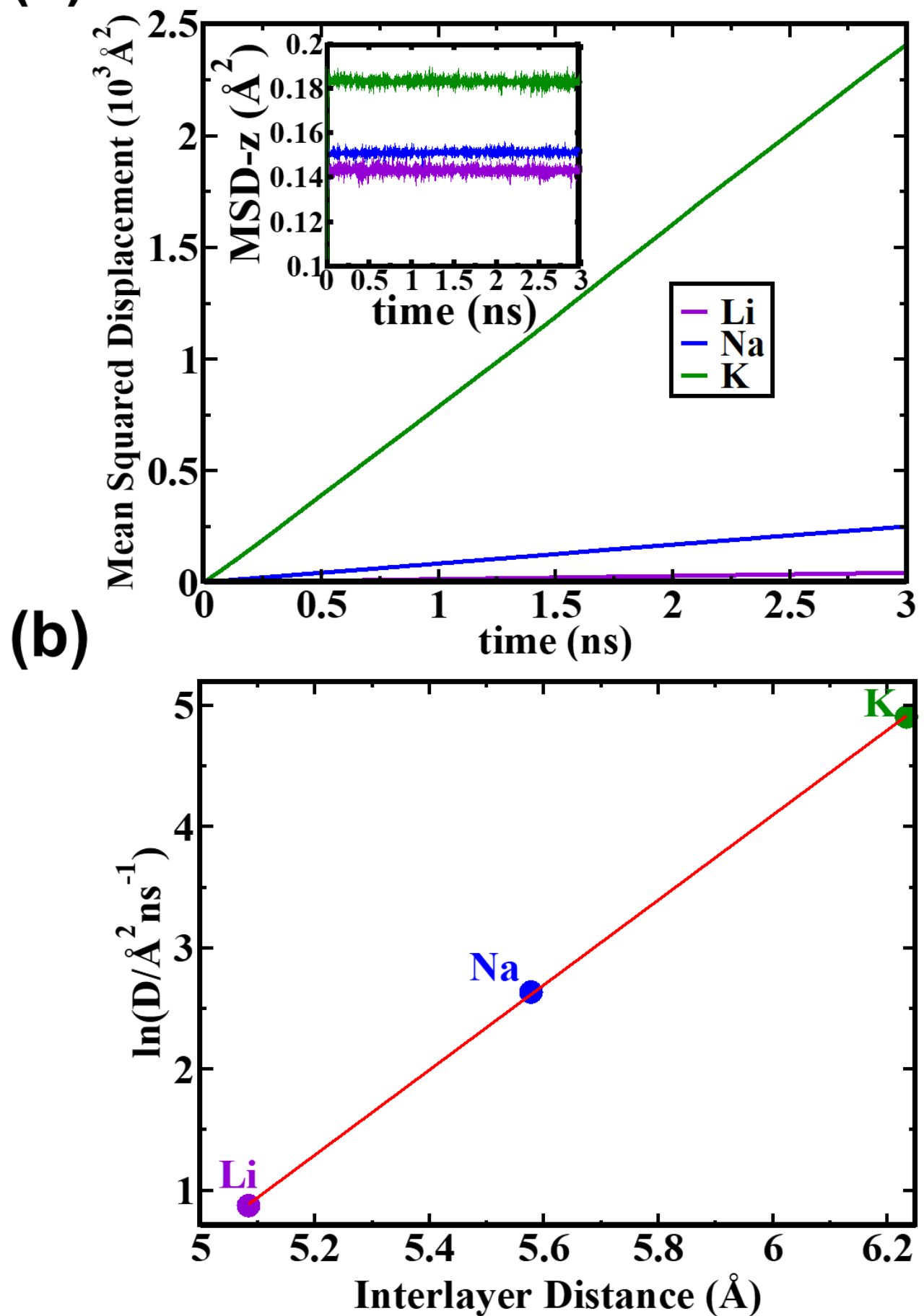

Figure 3. (a) The mean square displacement (MSD) of cations in $A_{2} \mathrm{Ni}_{2} \mathrm{TeO}_{6}(A=\mathrm{Li}$, $\mathrm{Na}$ and $\mathrm{K}$ ) based on isobaric-isothermal (NPT) MD simulations at $600 \mathrm{~K}$. Inset shows the MSD of cations (along the $c$-axis direction (MSD-z)). These results demonstrate that cationic mobility is restricted along the $a b$-plane, rendering the diffusion to be highly anisotropic. (b) A logarithmic plot of the diffusion coefficient $(D)$ as a function of the interlayer distance (calculated from the gradient of the MSD graphs in (a)). The linear fit interpolation is marked by a red line. 
The logarithm of diffusion coefficient $(D)$ versus inverse temperature $(1000 / T)$ is displayed in Figure 4(a). According to equation 3, $\ln D=\ln D_{0}-E a / k_{\mathrm{B}} T$ corresponds to the equation of a straight line, where $-E a / 1000 k_{\mathrm{B}} T$ is the slope and $\ln D_{0}$ the y-intercept. This implies that both the activation energy and the pre-exponential factor can be readily extracted from Figure 4(a). The activation energy of $\mathrm{Li}_{2} \mathrm{Ni}_{2} \mathrm{TeO}_{6}$ is found to be highest $(0.56 \mathrm{eV})$ and lowest $(0.26 \mathrm{eV})$ for $\mathrm{K}_{2} \mathrm{Ni}_{2} \mathrm{TeO}_{6}$. A comparison of the activation energies calculated from the present MD simulations and reported experimental results is shown in Table 3. The MD simulation results generally conform to the reported experimental results, although a slightly different value is obtained in the case of $\mathrm{Li}_{2} \mathrm{Ni}_{2} \mathrm{TeO}_{6}$. This is attributed to the difference in the crystallographic structure of $\mathrm{Li}_{2} \mathrm{Ni}_{2} \mathrm{TeO}_{6}$ compared to $\mathrm{Na}_{2} \mathrm{Ni}_{2} \mathrm{TeO}_{6}$ and $\mathrm{K}_{2} \mathrm{Ni}_{2} \mathrm{TeO}_{6}$. Nonetheless, the present MD simulation model successfully reproduces the diffusion trend observed in $\mathrm{Li}-, \mathrm{Na}-$ and K-based honeycomb layered nickel-based tellurate systems. The activation energy

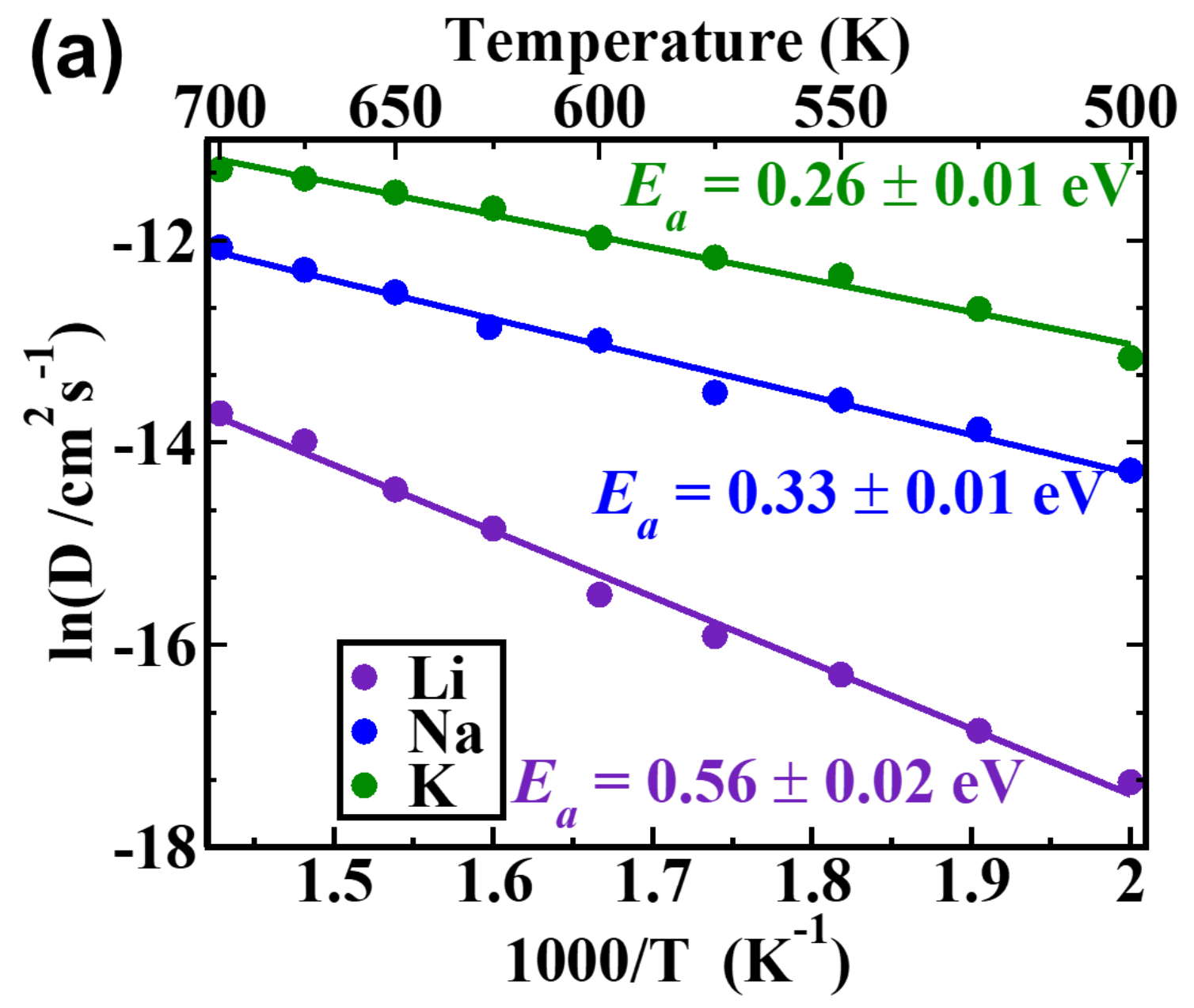




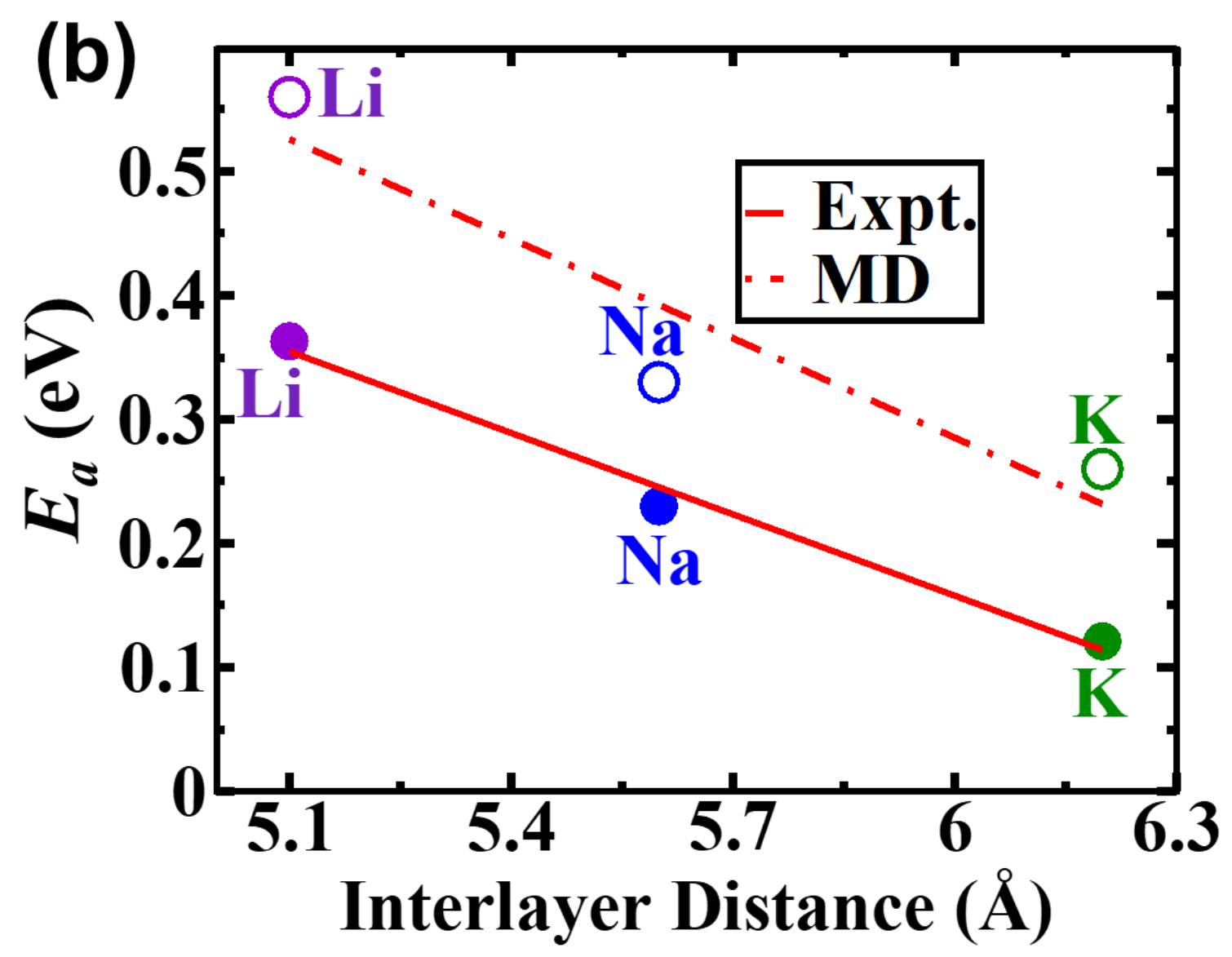

Figure 4. (a) Logarithmic plots of the diffusion coefficient $(D)$ for alkali ions in $A_{2} \mathrm{Ni}_{2} \mathrm{TeO}_{6}(A=\mathrm{Li}, \mathrm{Na}$ and $\mathrm{K}$ ) versus inverse temperature as extracted from molecular dynamics (MD) simulation results for the temperature range of 500-700 K at intervals of $25 \mathrm{~K}$. Errors in the attained activation energy values are determined from the standard deviation. The slopes for $\mathrm{Li}_{2} \mathrm{Ni}_{2} \mathrm{TeO}_{6}, \mathrm{Na}_{2} \mathrm{Ni}_{2} \mathrm{TeO}_{6}$ and $\mathrm{K}_{2} \mathrm{Ni}_{2} \mathrm{TeO}_{6}$ are respectively shown in purple, blue and green. (b) The activation energy values based on both experiment (abbreviated as Exp.) and computation (MD simulations) against the interlayer distances in $A_{2} \mathrm{Ni}_{2} \mathrm{TeO}_{6}(A=\mathrm{Li}, \mathrm{Na}$ and $\mathrm{K})$.

Table 3. Comparison of the activation energy values (in $\mathrm{eV}$ ) obtained from MD simulations with the reported experimental values.

\begin{tabular}{llllll}
\hline $\mathrm{Li}$ & & $\mathrm{Na}$ & & $\mathrm{K}$ & \\
$\mathrm{MD}$ & Expt. $^{[35]}$ & $\mathrm{MD}$ & Expt. & $\mathrm{MD}$ & Expt. $^{[27]}$ \\
\hline 0.530 & 0.363 & 0.330 & $0.207^{[35]}$, & 0.260 & 0.121 \\
& & & $0.230^{[20]}$ & & \\
\hline
\end{tabular}

values reported from both experimental and MD simulations display an almost linear 
decaying behaviour with increasing interlayer distances (as further shown in Figure 4(b)).

To comprehensively understand the influence of interlayer distances on the cationic diffusion of the present honeycomb layered oxides, it is paramount to isolate the interlayer distance from the cation size and treat them independently of each other even though they are typically correlated in real systems. Therefore, in the MD simulations, the $c$-parameter, which corresponds to the interlayer distance, is increased whilst the cation size is kept constant. The $c$-parameter was progressively increased from 11.1 to 13.1 $\AA$ at intervals of $0.2 \AA$, to cover the interlayer distance range from Li to $\mathrm{K}$. The ionic radius was kept constant at the value corresponding to that of $\mathrm{K}$ (i.e., $1.38 \AA$ ), as $\mathrm{K}$-ions have the largest ionic radius reported so far for this family of honeycomb layered oxides. As can be seen in the plot of the inter-slab distribution (furnished as Figure S1 in the Supplementary Information), increasing the $c$-parameter basically increases the inter-slab distance without forming other structural artifacts in the MD simulation, such as undulation. However, it is important to note that different size cations engender different interlayer distances to maintain structural stability. Thus, through MD simulations, it is possible to study microscopic phenomena beyond the reach of practical experiments.

Accordingly, the interlayer distances below the ionic radius of lithium (11 $\AA$ ) were not considered because lithium cations are the smallest cations that embody the present honeycomb layered framework. Moreover, the cationic diffusion was observed to diminish with decrease in the interlayer distances, as shown in Figure 5; indicative that interlayer distances smaller than $11 \AA$, would result in unfavourable cationic diffusion. Notably, the diffusivity trend significantly deviates from exponential dependency of interlayer distances as shown in Figure S2, whereby the logarithmic of diffusion coefficient plot shows a converging nature at higher interlayer distances. Although this might be a consequence of the current simulation conditions, where the $a$ and $b$ parameters and the ionic sizes are kept constant, it might also be an indicator that larger interlayer distances do not necessarily result in higher cationic diffusion presumably as a result of structural instability or diminished interlayer interactions. 


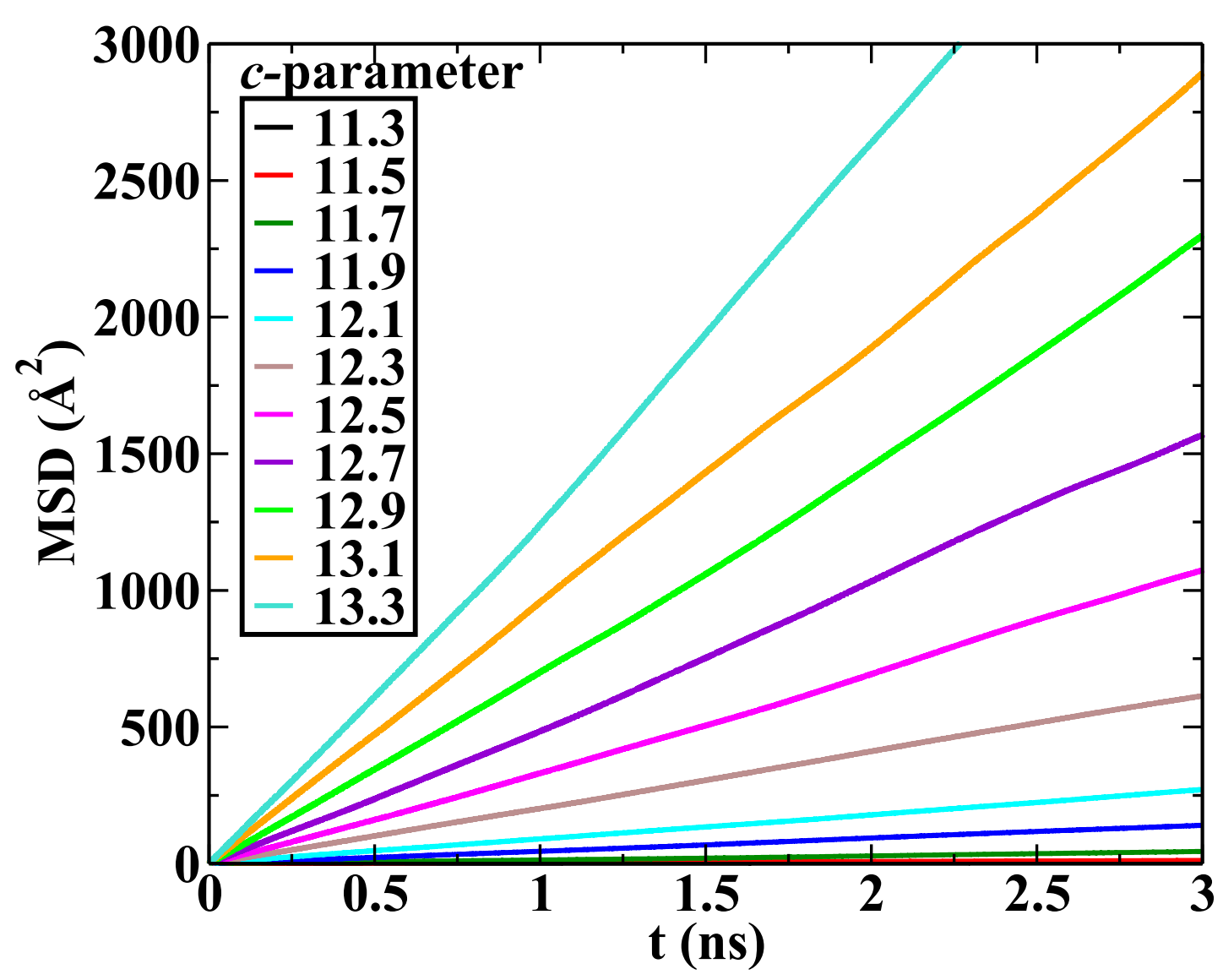

Figure 5. The mean square displacement (MSD) of cations in $\mathrm{K}_{2} \mathrm{Ni}_{2} \mathrm{TeO}_{6}$ based on isometric-isothermal (NVT) MD simulation at $600 \mathrm{~K}$, acquired by progressively increasing the $c$-parameter (from 11.3 to $13.3 \AA$ at intervals of $0.2 \AA$ ) with the cation size fixed to the size of $\mathrm{K}^{+}$ion. Note that the $11.3 \AA$ is almost superimposed on to the horizontal axis.

\subsection{Microscopic mechanisms of cationic transport}

Although the aforementioned results are already illuminating, it is imperative to take into consideration the atomistic mechanisms governing the cations confined within the sublattices along the $a b$-plane in order to accurately discern the nature of the enhanced diffusion in $\mathrm{Na}_{2} \mathrm{Ni}_{2} \mathrm{TeO}_{6}$ and $\mathrm{K}_{2} \mathrm{Ni}_{2} \mathrm{TeO}_{6}$ compared to $\mathrm{Li}_{2} \mathrm{Ni}_{2} \mathrm{TeO}_{6}$. On that account, three different cationic sites were identified from experimental studies inside the conduction plane of $A_{2} \mathrm{Ni}_{2} \mathrm{TeO}_{6}(A=\mathrm{Na}$ and $\mathrm{K})$ in each interlayer of a unit cell, labelled as $A 1, A 2$ and $A 3$, with a multiplicity of 3,2, and 1 respectively. ${ }^{[12,20,26,27]}$ (For clarity to readers, $A 1, A 2$ and $A 3$ are crystallographically distinct sites.) These sites are the centre of trigonal prisms formed by oxygen atoms, as shown in Figure 1. The octahedral 
layers include tetrahedral voids (or 'holes') formed between two neighbouring $\mathrm{NiO}_{6}$ and $\mathrm{TeO}_{6}$ octahedra with the $A 1$ site located mid-way between the two tetrahedral voids from the top and bottom layers. The $A 2$ sites are sandwiched between the triangular faces of $\mathrm{NiO}_{6}$ octahedra from the top and bottom layers, whereas the $A 3$ site is located between two $\mathrm{TeO}_{6}$ octahedra. However, the experimentally reported $\mathrm{Li}_{2} \mathrm{Ni}_{2} \mathrm{TeO}_{6}$ shows a different structure whereby the $\mathrm{NiO}_{6}$ octahedra are slightly deformed, and the layered stacking sequence is different from the $\mathrm{Na}_{2} \mathrm{Ni}_{2} \mathrm{TeO}_{6}$ and $\mathrm{K}_{2} \mathrm{Ni}_{2} \mathrm{TeO}_{6}$ which have the $\mathrm{TeO}_{6}$ octahedra at the top and bottom of the $A 3$-sites. The smaller interlayer separation (5.08 $\AA$ ) in $\mathrm{Li}_{2} \mathrm{Ni}_{2} \mathrm{TeO}_{6}$ (compared to $\mathrm{Na}_{2} \mathrm{Ni}_{2} \mathrm{TeO}_{6}(5.57 \AA)$ and $\mathrm{K}_{2} \mathrm{Ni}_{2} \mathrm{TeO}_{6}(6.23 \AA)$ ), engenders a high $\mathrm{Te}-\mathrm{Te}$ Coulombic repulsion along the $c$-axis direction which prevents the $\mathrm{TeO}_{6}$ octahedra from achieving a stable vertical orientation. Thus, unlike the $\mathrm{Na}$ and $\mathrm{K}$ systems, the Li cations orient themselves at the top and bottom of the octahedral voids in order to avoid the resultant strong repulsion at the $A 3$ site.

In order to understand cationic site preferences and hopping energy barriers between sites, the population density pattern and average potential energy of the mobile cations were calculated in accordance with equation 5 (detailed in the METHODOLOGY section). Both the population density and potential energy are projected onto a $2 \mathrm{D}$ grid on the $a b$-plane (with all the coordinates of the cations folded into a single cell). Figure 6 displays the population density, whilst Figure 7 shows the corresponding potential energy surface of cations in the order of $\mathrm{Li}, \mathrm{Na}$ and $\mathrm{K}$ (replicated over $2 \times 2$-unit cells for easy visualisation of their continuity). The Li population densities are mostly localised due to the deep potential wells with minima of $-3.2 \mathrm{eV}$ (Figures 6a and 7a), whereas the Na-substituted system exhibits well-connected cationic diffusion channels between the $A 1$ and $A 2$ sites (Figures $\mathbf{6 b}$ and $\mathbf{7 b}$ ). Due to the strong repulsion previously mentioned, there are no $\mathrm{Na}$ cations occupying the $A 3$ sites which are located directly 

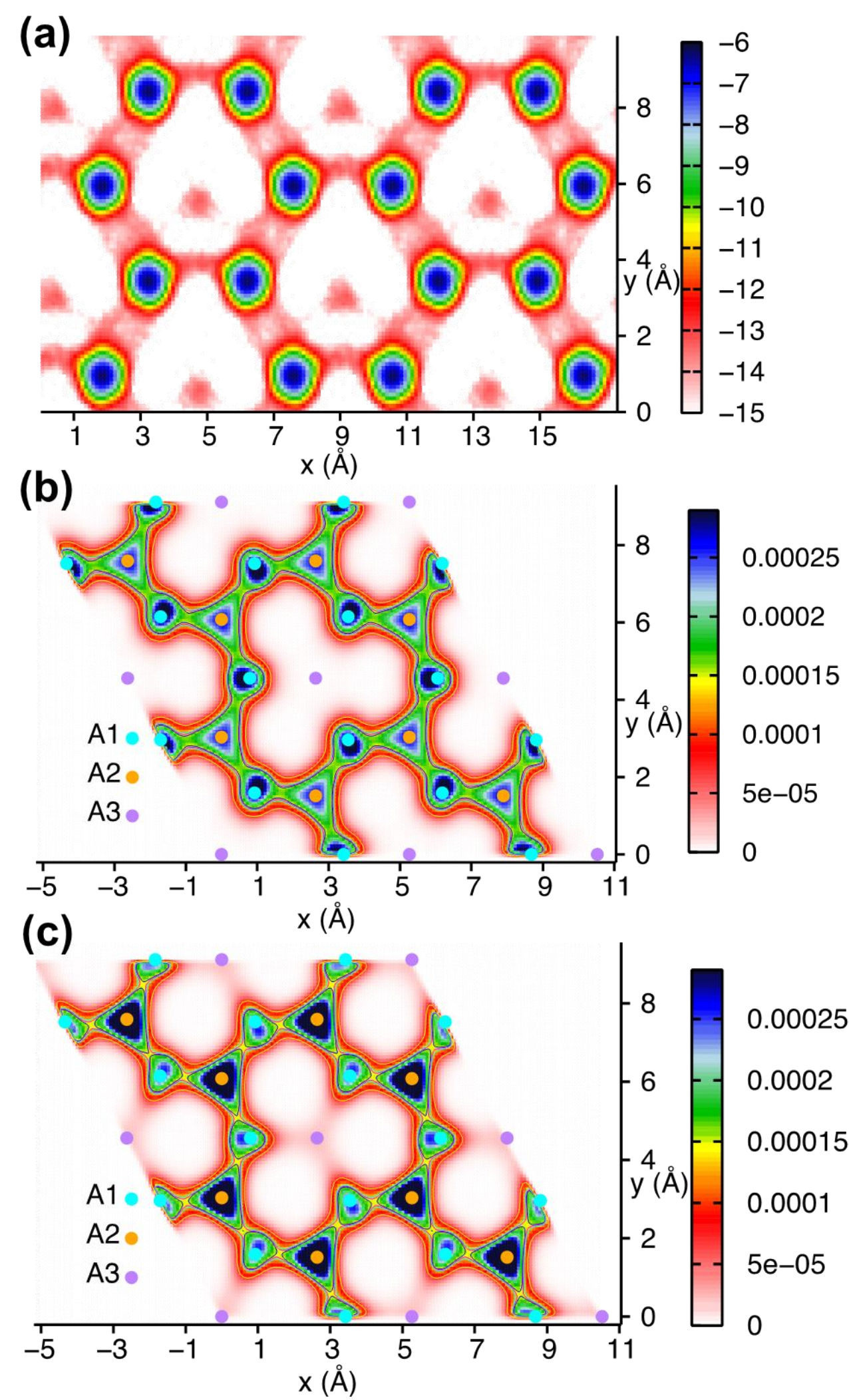

Figure 6. Average individual population profiles of mobile $A$ cations for $A_{2} \mathrm{Ni}_{2} \mathrm{TeO}_{6}$ $(A=\mathrm{Li}$, Na and $\mathrm{K}$ ) derived from NVT-MD simulations at $600 \mathrm{~K}$ (mapped on to $2 \times 2$-unit cells). The population profiles for (a) $\mathrm{Li}_{2} \mathrm{Ni}_{2} \mathrm{TeO}_{6}$, (b) $\mathrm{Na}_{2} \mathrm{Ni}_{2} \mathrm{TeO}_{6}$ and (c) $\mathrm{K}_{2} \mathrm{Ni}_{2} \mathrm{TeO}_{6}$. Note that the colour bar in (a) in represented in a logarithmic scale for better visualisation. A common colour bar is used in both (b) and (c). The location of the crystallographically distinct alkali atom sites for $\mathrm{Na}_{2} \mathrm{Ni}_{2} \mathrm{TeO}_{6}$ and $\mathrm{K}_{2} \mathrm{Ni}_{2} \mathrm{TeO}_{6}$, namely 
$A 1, A 2$, and $A 3$, are shown in legends in (b) and (c). The population contours reflect that the preferred migration pathway of the cations is solely within the $A 1$ and $A 2$ sites (viz., $A 1-A 2-A 1-A 2 \ldots)$. Note that the $\mathrm{Li}$ sites in $\mathrm{Li}_{2} \mathrm{Ni}_{2} \mathrm{TeO}_{6}$ are not marked, as their population density at $600 \mathrm{~K}$ deviates from that at room temperature in their crystallographic sites.

between the top and bottom $\mathrm{Te}-\mathrm{Te}$ octahedra along the $c$-axis direction. In the case of $\mathrm{Na}_{2} \mathrm{Ni}_{2} \mathrm{TeO}_{6}$, the potential wells are relatively shallow $(-2.7 \mathrm{eV})$ compared to the $\mathrm{Li}$ system. Conversely, $\mathrm{K}_{2} \mathrm{Ni}_{2} \mathrm{TeO}_{6}$ exhibits a slightly different population profile (Figures 6c and 7c), characterised by the shallowest potential wells $(-2.3 \mathrm{eV})$ amongst the three systems. This attests to the considerably the low hopping energy barrier of $\mathrm{K}$ allowing more facile shifts of $\mathrm{K}$ cations from the $A 1$ sites to the $A 2$ site compared to the $\mathrm{Na}$ system. These results therefore evince that the minima of the potential energy increase in the order of $\mathrm{Li}, \mathrm{Na}$ and $\mathrm{K}$, which also corresponds to increasing order of the interlayer distances, indicative that the energy barrier inhibiting the hopping of the cations between sites is reduced with inter-layer distance leading to a drastically higher cationic diffusion. This was additionally affirmed by calculations of the cationic occupancy at the $A 1$ and $A 2$ sites in relation to the progressively increasing interlayer distances as shown in Figure S3. A similar trend entailing a progressive decrease in occupancy at the $A 1$ site with increasing interlayer distances, concomitantly lifting the energy minima at the $A 2$-site.

For a detailed quantitative estimation of the occupancy and nature of cationic hopping energy barrier, a 2D plot of population distribution and free energy distribution was calculated from the population distribution along a straight-line path conjured up by an imaginary cylindrical axis with a radius of $0.8 \AA$ connecting the neighbouring $A 2$ and $A 1$ sites in accordance with equation 6 (see METHODOLOGY section). In the $\mathrm{Na}$ and $\mathrm{K}$ systems, a slightly different $A 1$ and $A 2$ site occupancy is revealed by the $2 \mathrm{D}$ population density plot, as shown respectively in Figures $\mathbf{6 b}$ and $\mathbf{6 c}$. In the Na system, the population densities at the $A 1$ and $A 2$-sites are almost identical whereas in the $\mathrm{K}$ system, the population at the $A 2$-sites is significantly higher than the $A 1$-sites. Remarkably, free energy distribution calculations from the respective population density distributions revealed that although $\mathrm{K}$ system exhibits a lower activation energy than the $\mathrm{Na}$ system (Figures $\mathbf{7 b}$ and $\mathbf{7 c}$ ), the free energy barrier for $\mathrm{K}$ ion diffusion $(0.07 \mathrm{eV})$ is higher than its $\mathrm{Na}$ counterpart $(0.04 \mathrm{eV})$. This juxtaposed behaviour can be ascribed to the site topology and the connectivity of the two systems. As illustrated in Figure 6 ((b) 


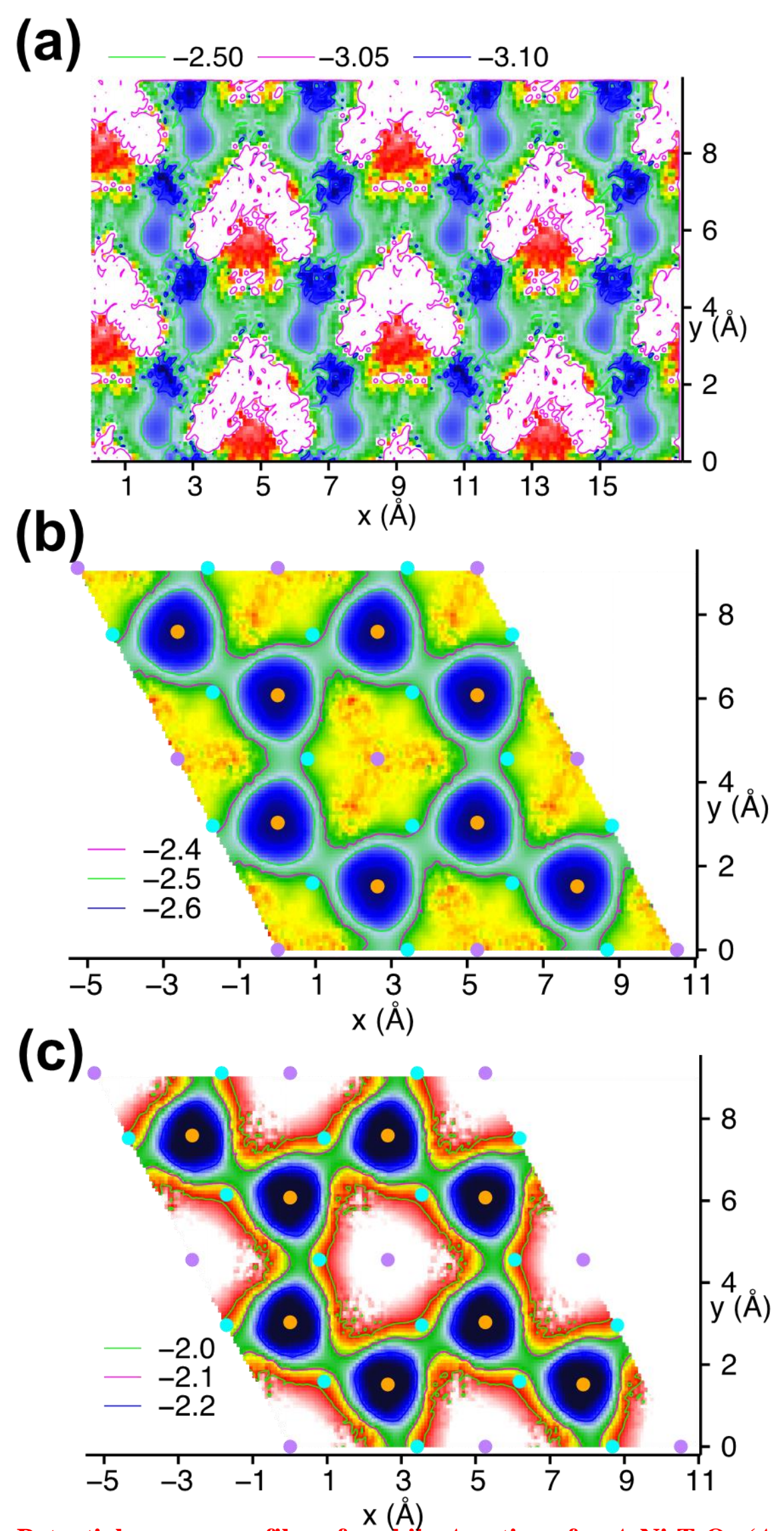

Figure 7. Potential energy profiles of mobile $A$ cations for $A_{2} \mathrm{Ni}_{2} \mathrm{TeO}_{6}(A=\mathrm{Li}$, Na and $\mathrm{K}$ ) derived from NVT-MD simulations at $600 \mathrm{~K}$ (mapped on to $2 \times 2$-unit cells). The potential energy profiles for (a) $\mathrm{Li}_{2} \mathrm{Ni}_{2} \mathrm{TeO}_{6}$, (b) $\mathrm{Na}_{2} \mathrm{Ni}_{2} \mathrm{TeO}_{6}$ and (c) $\mathrm{K}_{2} \mathrm{Ni}_{2} \mathrm{TeO}_{6}$. The location of the crystallographically distinct alkali atom sites for $\mathrm{Na}_{2} \mathrm{Ni}_{2} \mathrm{TeO}_{6}$ and $\mathrm{K}_{2} \mathrm{Ni}_{2} \mathrm{TeO}_{6}$, namely $A 1, A 2$, and $A 3$, are shown in legends in (b) and (c). 
and (c)) and Figure 7((b) and (c)), the $A 1$ sites are connected by two $A 2$ sites, whereas $A 2$ sites are connected by three $A 1$ sites implying that a cation occupying an $A 1$ site has two possible paths, whereas it finds three possible paths if it occupies an A2-site. Therefore, because the Na-system is characterised by higher population densities at the A1-sites, cationic diffusion will be restricted by the lower availability of paths. On the other hand, the higher population $\mathrm{K}$ ions at the $A 2$-sites provides more avenues for cationic diffusion despite the higher free energy barrier (see Figure S4 in the Supplementary Information). This postulation is further corroborated by the population density distribution trends that show higher occupation of $A 2$ site with increasing interlayer distances.

For a deeper understanding of the role of the interlayer distance in cationic diffusion at an atomistic level, the bottleneck radius formed by the oxygen atoms, was calculated along the cationic diffusion pathway. As defined by Evstigneeva and co-workers, ${ }^{[20]}$ the bottleneck radius is the smallest $A-\mathrm{O}$ distance at the saddle point in the cationic diffusion path from the $A 1$ to $A 2$ sites, as shown in Figure 8. In the present study, the other bottlenecks formed between the path $A 1$ to $A 3$ are not taken into account, as they do not play any role on cationic diffusion owing to the cationic absence at the $A 3$-sites. The results indicate that the bottleneck distance for the Na-system $(2.35 \AA)$ is smaller than the K-system $(2.57 \AA)$ as is evident in Figure 9. Thus, the Na system embodies a stronger force of attraction at the saddle points of the free energy path owing to the closer $\mathrm{O}$ atoms, effectively inhibiting cationic diffusion along the channels. Conversely, the $\mathrm{K}$ system manifests the weakest force of attraction at the saddle points due to the distant $\mathrm{O}$ atoms, yielding the highest cationic diffusion rate as observed in the MD simulation results. Figure 9 indeed depicts a similar trend. That is, larger interlayer distances confer wider bottleneck radii; thus, resulting in higher cationic diffusion, as further displayed in Figure 5. By varying ionic radius whilst keeping the interlayer fixed, the effective bottleneck radius increases because of smaller size cation. Thus, the smaller size cation can easily pass through the same size bottleneck, resulting in higher cationic diffusion (as evident in Figure S5). A similar behaviour was also reported in other materials, $\mathrm{Li} M_{2}\left(\mathrm{PO}_{4}\right)_{3}$ (where $M=\mathrm{Zr}$, Hf, Sn and Ti) ${ }^{[36]}$ where larger bottleneck radii formed by oxygen atoms facilitates the cationic diffusion. The accurate depiction of the progressive increase in the bottleneck radius with increasing interlayer distances, affirms the use of MD simulations as an auspicious tool to decipher the atomistic mechanisms of honeycomb layered oxides. 


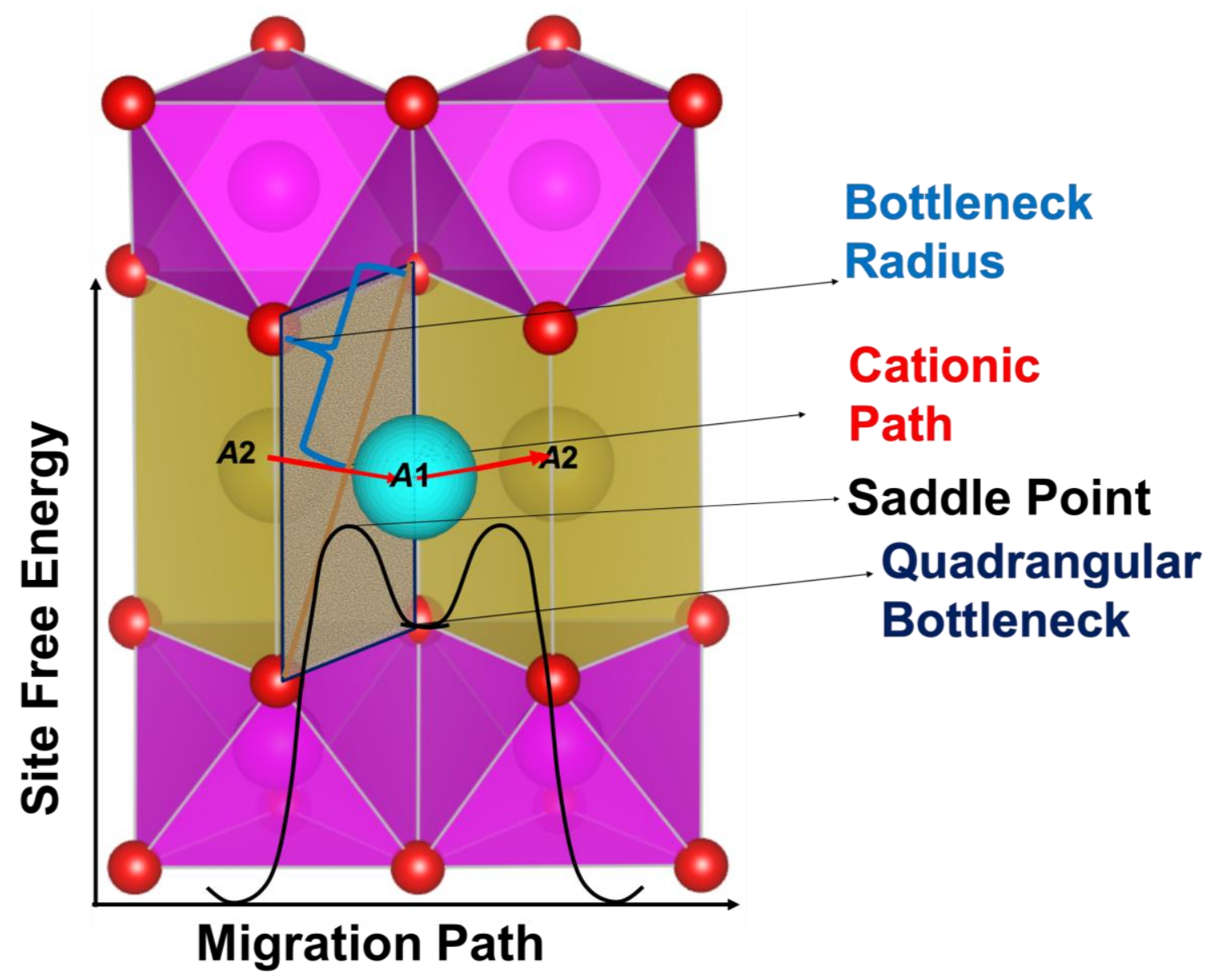

Figure 8. Schematic representation of the cation-hopping path from $A 2$ to $A 1$ sites across the $A_{2} \mathrm{Ni}_{2} \mathrm{TeO}_{6}$ topology and the associated free energy landscape. The bottleneck radius and the cationic migration path have been highlighted. A curve illustrating the free-energy landscape is also appended, for clarity. The quadrangular bottleneck is located near the transition state (one of the bottleneck radii is shaded for emphasis). Different colours have been used for atoms residing in $A 1$ and $A 2$ sites, for clarity. 


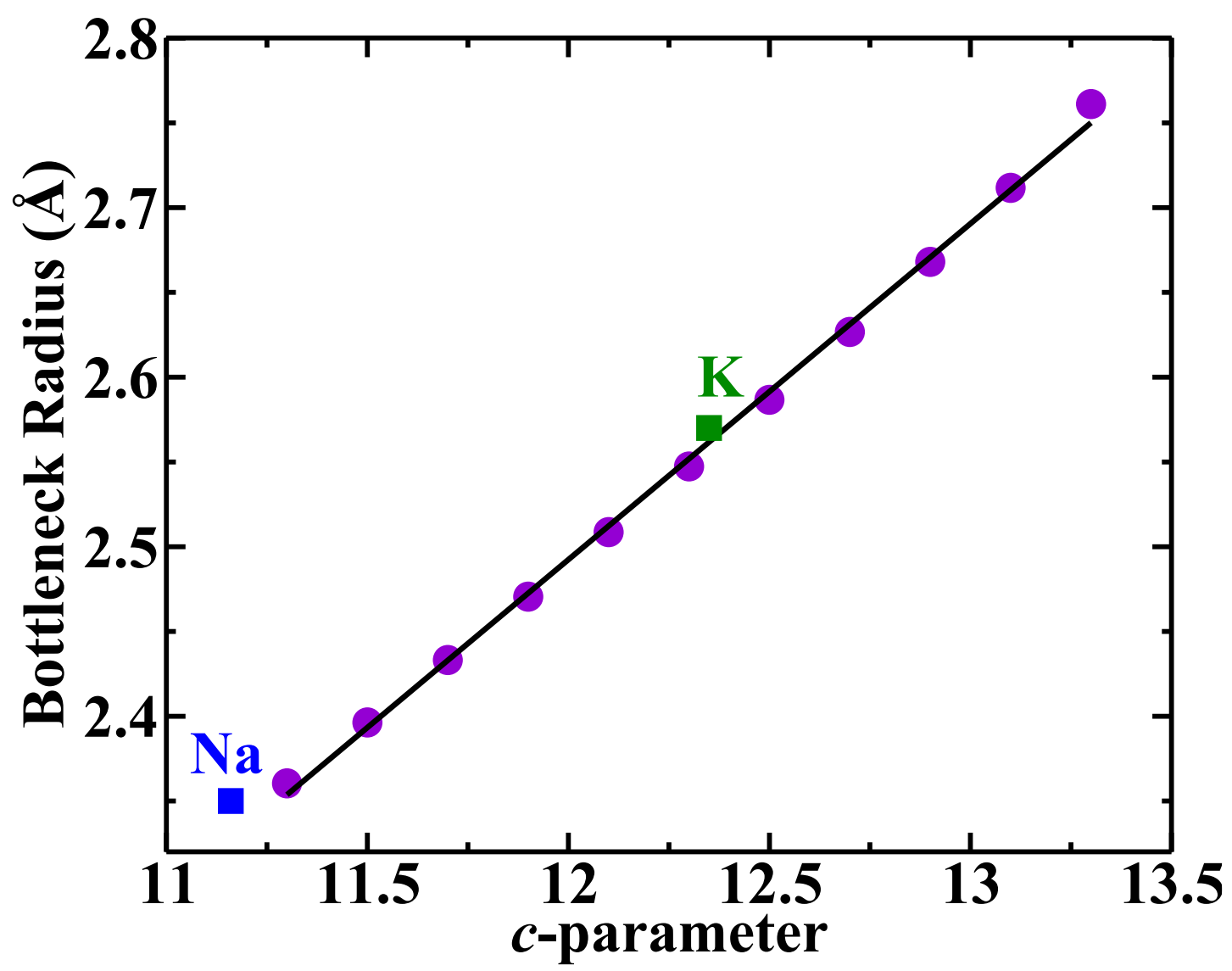

Figure 9. Bottleneck radius formed by oxygen atoms located between the cationic path from $A 1$ to $A 2$ for the different $c$-parameters. The bottleneck radii for $\mathrm{Na}$ and $\mathrm{K}$ are marked using blue and green square symbols, respectively.

In light of the observations presented in this study, it can be deduced that chemical compositions of $A_{2} \mathrm{Ni}_{2} \mathrm{TeO}_{6}$ entailing lithophile atoms with larger ionic sizes than $\mathrm{K}$ such as $A=\mathrm{Rb}$ or $\mathrm{Cs}$ could be a propitious strategy for enhancing the cationic diffusion for honeycomb layered oxide family discussed herein. However, MD simulations suggest that such systems are relatively unstable, because of the extremely weak Van der Waal interactions between adjacent layers.

\section{CONCLUSION}

In this study, we present systematic molecular dynamics (MD) simulations using a refined set of Vashishta-Rahman form of interatomic potential parameters of the cations in $A_{2} \mathrm{Ni}_{2} \mathrm{TeO}_{6}$ (where $A=\mathrm{Li}, \mathrm{Na}$, and $\mathrm{K}$ ) family of honeycomb layered oxides. The MD 
simulations successfully reproduce the correct structure, conductivity and population patterns of the cations residing at different crystallographic sites, in conformity with previously reported experimental results. ${ }^{[26-28]}$ Our observations indicate that the cationic diffusion increases exponentially with interlayer distance. Further, we ascertain that the enhanced cationic diffusion behaviour arises from the weak interactions between the cations and the adjacent layers of the material as the interlayer distance is progressively increased in the simulation. Even taking into account the potential energy landscape of the sublattices, similar observations are reproduced whereby the cationic hopping energy barrier inhibiting effective diffusion increases in the order of $\mathrm{Li}, \mathrm{Na}$ and $\mathrm{K}$ systems which correspond to the bottleneck radii in the alkali diffusion channel. Moreover, the occupancy of the atoms (in crystallographically different sites) varies with increasing interlayer distances, in favour of larger accessible cationic pathways. The results reported herein not only affirm the remarkable performance of honeycomb layered oxides but also validate the use of computation techniques such as molecular dynamic simulations as a propitious path in the quest of high-performance materials for future capacious batteries.

\section{ACKNOWLEDGEMENT}

We gratefully acknowledge the Center for Computational Materials Science of Institute for Materials Research, Tohoku University for permitting the use of MASAMUNEIMR (MAterial science Supercomputing system for Advanced MUltiscale simulations toward NExt-generation Institute of Material Research) (project no. 19S0010). This work was conducted in part under the auspices of the Japan Society for the Promotion of Science (JSPS KAKENHI Grant Number 19K15685), National Institute of Advanced Industrial Science and Technology (AIST) and Japan Prize Foundation.

\section{SUPPLEMENTARY MATERIAL}

The online link to Supplementary material (which includes details regarding the computational methodology) associated with this article will be availed at the production stage. 


\section{REFERENCES}

1. Manthiram, A. A reflection on lithium-ion battery cathode chemistry. Nat. Commun. 11, 1550 (2020).

2. Nitta, N., Wu, F., Lee, J. T. \& Yushin. G. Li-ion battery materials: present and future. Mater. Today 18, 252-264 (2015).

3. Armand, M. \& Tarascon, J. -M. Building Better Batteries. Nature 451, 652-657 (2008).

4. Hosaka, T., Kubota, K., Hameed, A. S. \& Komaba, S. Research Development on K-Ion Batteries. Chem. Rev. 120, 6358-6466 (2020).

5. Yabuuchi, N., Kubota, K., Dahbi, M. \& Komaba, S. Research Development on Sodium-Ion Batteries. Chem. Rev. 114, 11636-11682 (2014).

6. Hironaka, Y., Kubota, K. \& Komaba, S. P2- and P3- $\mathrm{K}_{x} \mathrm{CoO}_{2}$ as an electrochemical potassium intercalation host. Chem. Commun. 53, 3693-3696 (2017).

7. Kanyolo, G. M. et al. Honeycomb Layered Oxides: Structure, Energy Storage, Transport, Topology and Relevant Insights. arXiv. arXiv:2003.03555 (2020).

8. Sathiya, M. et al. $\mathrm{Li}_{4} \mathrm{NiTeO}_{6}$ as a positive electrode for Li-ion batteries. Chem. Commun. 49, 11376-11378 (2013).

9. Grundish, N. S., Seymour, I. D., Henkelman, G. \& Goodenough J. B. Electrochemical Properties of Three $\mathrm{Li}_{2} \mathrm{Ni}_{2} \mathrm{TeO}_{6}$ Structural Polymorphs. Chem. Mater. 31, 9379-9388 (2019).

10. Yang, Z. et al. A high-voltage honeycomb-layered $\mathrm{Na}_{4} \mathrm{NiTeO}_{6}$ as cathode material for Na-ion batteries. J. Power Sources 360, 319-323 (2017).

11. Yuan, D. et al. A Honeycomb-Layered $\mathrm{Na}_{3} \mathrm{Ni}_{2} \mathrm{SbO}_{6}$ : A High-Rate and Cycle-Stable Cathode for Sodium-Ion Batteries. Adv. Mater. 26, 6301-6306 (2014).

12. Masese, T. et al. Rechargeable potassium-ion batteries with honeycomb-layered tellurates as high voltage cathodes and fast potassium-ion conductors. Nat. Commun 9, 3823 (2018).

13. Masese, T. et al. A high voltage honeycomb layered cathode framework for rechargeable potassium-ion battery: P2-type $\mathrm{K}_{2 / 3} \mathrm{Ni}_{1 / 3} \mathrm{Co}_{1 / 3} \mathrm{Te}_{1 / 3} \mathrm{O}_{2}$. Chem. Commun. (Camb) 55, 985-988 (2019).

14. Yoshii, K. et al. Sulfonylamide-Based Ionic Liquids for High-Voltage Potassium-Ion Batteries with Honeycomb Layered Cathode Oxides. ChemElectroChem 6, 3901-3910 (2019). 
15. Chen, C. -Y. et al. High-voltage honeycomb layered oxide positive electrodes for rechargeable sodium batteries. Chem. Commun. (Camb) 56, 9272-9275 (2020).

16. Bhange, D. S. et al. Honeycomb-layer structured $\mathrm{Na}_{3} \mathrm{Ni}_{2} \mathrm{BiO}_{6}$ as a high voltage and long life cathode material for sodium-ion batteries. J. Mater. Chem. A 5, 1300-1310 (2017).

17. Zheng, L. \& Obrovac, M. N. Honeycomb Compound $\mathrm{Na}_{3} \mathrm{Ni}_{2} \mathrm{BiO}_{6}$ as Positive Electrode Material in Na Cells. J. Electrochem. Soc. 163, A2362-A2367 (2016).

18. Ma, J. et al. Ordered and Disordered Polymorphs of $\mathrm{Na}\left(\mathrm{Ni}_{2 / 3} \mathrm{Sb}_{1 / 3}\right) \mathrm{O}_{2}$ : Honeycomb-Ordered Cathodes for Na-Ion Batteries. Chem. Mater. 27, 2387-2399 (2015).

19. Gupta, A., Mullins, G. B. \& Goodenough, J. B. $\mathrm{Na}_{2} \mathrm{Ni}_{2} \mathrm{TeO}_{6}$ : Evaluation as a cathode for sodium battery. J. Power Sources 243, 817-821 (2013).

20. Evstigneeva, M. A., Nalbandyan, V. B., Petrenko, A. A., Medvedev, B. S. \& Kataev, A. A. A new family of fast sodium ion conductors: $\mathrm{Na}_{2} \mathrm{M}_{2} \mathrm{TeO}_{6}(\mathrm{M}=\mathrm{Ni}, \mathrm{Co}, \mathrm{Zn}$, Mg). Chem. Mater. 23, 1174-1181 (2011).

21. Berthelot, R., Schmidt, W., Sleight, A. W. \& Subramanian, M. A. Studies on solid solutions based on layered honeycomb-ordered phases $\mathrm{P} 2-\mathrm{Na}_{2} \mathrm{M}_{2} \mathrm{TeO}_{6}(\mathrm{M}=\mathrm{Co}, \mathrm{Ni}$, Zn). J. Solid State Chem. 196, 225-231 (2012).

22. Yadav, D. K., Sethi, A., Shalu \& Uma, S. New series of honeycomb ordered oxides, $\mathrm{Na}_{3} \mathrm{M}_{2} \mathrm{SbO}_{6}(\mathrm{M}(\mathrm{ii})=\mathrm{Mn}, \mathrm{Fe},(\mathrm{Mn}, \mathrm{Fe}),(\mathrm{Mn}, \mathrm{Co}))$ : Synthesis, structure and magnetic properties. Dalt. Trans. 48, 8955-8965 (2019).

23. Nalbandyan, V. B., Petrenko, A. A. \& Evstigneeva, M. A. Heterovalent substitutions in $\mathrm{Na}_{2} \mathrm{M}_{2} \mathrm{TeO}_{6}$ family: Crystal structure, fast sodium ion conduction and phase transition of $\mathrm{Na}_{2} \mathrm{LiFeTeO}_{6}$. Solid State Ionics 233, 7-11 (2013).

24. Schmidt, W., Berthelot, R., Etienne, L., Wattiaux, A. \& Subramanian, M. A. Synthesis and characterization of $\mathrm{O} 3-\mathrm{Na}_{3} \mathrm{LiFeSbO}_{6}$ : A new honeycomb ordered layered oxide. Mater. Res. Bull. 50, 292-296 (2014).

25. Kumar, V., Bhardwaj, N., Tomar, N., Thakral, V. \& Uma, S. Novel lithium-containing honeycomb structures. Inorg. Chem. 51, 10471-10473 (2012).

26. Karna, S. K. et al. Sodium layer chiral distribution and spin structure of $\mathrm{Na}_{2} \mathrm{Ni}_{2} \mathrm{TeO}_{6}$ with a Ni honeycomb lattice. Phys. Rev. B 95, 104408 (2017).

27. Matsubara, N. et al. Magnetism and ion diffusion in honeycomb layered oxide $\mathrm{K}_{2} \mathrm{Ni}_{2} \mathrm{TeO}_{6}$. Sci. Rep. 10, 18305 (2020).

28. Bera, A. K. \& Yusuf, S. M. Temperature-Dependent Na-Ion Conduction and Its Pathways in the Crystal Structure of the Layered Battery Material $\mathrm{Na}_{2} \mathrm{Ni}_{2} \mathrm{TeO}_{6}$. Phys. Rev. B 95, 104408 (2017). 
29. Kumar, P. P. \& Yashonath, S. Ionic conduction in the solid state. J. Chem. Sci. 118, 135-154 (2006).

30. Sau, K. \& Kumar, P. P. Ion Transport in $\mathrm{Na}_{2} \mathrm{M}_{2} \mathrm{TeO}_{6}$ : Insights from Molecular Dynamics Simulation. J. Phys. Chem. C 119 1651-1658 (2015).

31. Sau, K. \& Kumar, P. P. Role of Ion-Ion Correlations on Fast Ion Transport: Molecular Dynamics Simulation of $\mathrm{Na}_{2} \mathrm{Ni}_{2} \mathrm{TeO}_{6}$. J. Phys. Chem. C 119 18030-18037 (2015).

32. Sau, K. Influence of ion-ion correlation on $\mathrm{Na}^{+}$transport in $\mathrm{Na}_{2} \mathrm{Ni}_{2} \mathrm{TeO}_{6}$ : molecular dynamics study. Ionics 22 2379-2385 (2016).

33. Sau, K., Ikeshoji, T. \& Roy, S. Role of divalent cation (Ba) substitution in the Li+ ion conductor $\mathrm{LiTi}_{2}\left(\mathrm{PO}_{4}\right)_{3}$ : a molecular dynamics study. Phys. Chem. Chem. Phys. 22 14471-14479 (2020).

34. Vashishta, P. \& Rahman,A. Ionic Motion in $\alpha$-AgI. Phys. Rev. Lett. 401337 (1978).

35. Zubayer, A. Ion Dynamics \& Magnetic Order in 2D Honeycomb Materials. DOI: 10.13140/RG.2.2.25447.06567 (2020).

36. Pramanik, K., Sau, K. \& Kumar, P. P. Role of Framework Flexibility in Ion Transport: A Molecular Dynamics Study of $\mathrm{LiM}_{2}{ }^{\mathrm{IV}}\left(\mathrm{PO}_{4}\right)_{3}$. J. Phys. Chem. C 124 4001-4009 (2020). 